\title{
Primeiro modelo matemático da cosmologia: as esferas concêntricas de eudoxo
}

First mathematical model of cosmology: the concentric spheres of eudoxus

\author{
Alan Miguel Velásquez-Toribio*1잉, Marcos Venicios Oliveira ${ }^{10}$ \\ ${ }^{1}$ Universidade Federal do Espirito Santo, Departamento de Física, Vitória, ES, Brasil.
}

Recebido em 05 de Abril, 2018. Revisado em 24 de Agosto, 2018. Aceito em 25 de Agosto, 2018.

\begin{abstract}
O modelo cosmológico de Eudoxo de Cnido (408 - 355 a.C.), o modelo das esferas concêntricas, representa o primeiro modelo matemático da cosmologia, o qual tenta explicar o movimento dos corpos celestes. Através dos comentários de Aristóteles (384 - 322 a.C.), dos escritos de Simplício (490 - 560 d.C.) e das abordagens feitas por historiadores e matemáticos do século XIX, será apresentada a reconstrução matemática clássica deste modelo. Também utilizamos um método matemático moderno, o método das matrizes de rotação, para ilustrar os movimentos planetários que resultam do modelo de Eudoxo, e determinar a equação paramétrica da hipópede. Devido à inexistência dos registros históricos originais do modelo, é necessário abordar as principais críticas a esta reconstrução clássica do século XIX, entre elas, a unicidade da reconstrução do modelo. No entanto, mesmo com todas as incertezas na reconstrução, ao longo dos séculos, o modelo de Eudoxo se apresenta como a primeira tentativa de entender, com as observações e as ferramentas da matemática da época, os movimentos do Sol, da Lua e os movimentos retrógrados dos planetas, e este trabalho dedica-se a discutir estas características de forma ampla, esgotando as principais obras apresentadas na literatura.
\end{abstract}

Palavras-chave: Esferas concêntricas. Cosmologia. Planetas. Hipópede de Eudoxo.

The cosmological model of Eudoxus of Cnidus (408 - 355 BC), the concentric spheres model, represents the first mathematical model of cosmology, which attempts to explain the motion of celestial bodies. Through the comments of Aristotle (384-322 BC), the writings of Simplicius (490-560 AD) and the approaches made by 19th century historians and mathematicians, the classical mathematical reconstruction of this model will be presented. We also use a modern mathematical method, the rotation matrix method, to illustrate the planetary motions that result from the Eudoxus model, and to determine the parametric equation of the hippopede. Due to the inexistence of the original historical records of the model, it is necessary to consider the main criticisms of this classic reconstruction of the nineteenth century, among them, the uniqueness of the reconstruction of the model. However, even with all the uncertainties in the reconstruction, over the centuries, the Eudoxus model presents itself as the first attempt to understand, with the observations and tools of mathematics of the time, the movements of the Sun, the Moon and the movements retrograde of the planets, and this work is dedicated to discuss these characteristics broadly, exhausting the main works presented in the literature.

Keywords: Concentric spheres, Cosmology, Planets, Hippopede of Eudoxus.

\section{Introdução}

Os Gregos no século V a.C., avançaram consideravelmente em observações astronômicas, pois entre outras razões, tinham fontes importantes e antigas de conhecimentos astronômicos das culturas Mesopotâmicas e Egípcias. Fazer um balanço do conjunto de conhecimentos astronômicos gregos no século $\mathrm{V}$ é muito complicado pois as evidências registradas são muito esparsas. Quando se observam os registros astronômicos antigos nota-se poucas fontes para reconstruir a história astronômica da época, inclusive historiadores como Neugebauer (1899 1990) comenta este fato comparando a grande quantidade de registros mesopotâmicos em comparação com os regis-

*Endereço de correspondência: alan.toribio@ufes.br. tros grego $\$^{1}$, 1, 2. No entanto, pesquisas recentes como os estudos da máquina de Anticítera [3] tem mostrado como a influência babilônica era forte na antiga Grécia, inclusive no século II a.C. 2

No geral, é possível afirmar que o desenvolvimento inicial da Astronomia estava associada com questões práticas como o trabalho da agricultura, da construção de templos e para marcar datas de rituais religiosos relacionados em muitos casos com mitos cosmológicos, entre outros. Por exemplo, os babilônios construíram grandes registros astronômicos que incluem séculos de

${ }^{1}$ Especificamente ver o artigo de Neugebauer, On some aspects of Early Greek Astronomy, incluido na referência mencionada [1,2]. ${ }^{2}$ Evans e Carman compararam as predições de eclipses da máquina de Anticítera com a predições babilônicas concluindo que a máquina data de 205 a.C., e se observam fortes evidências que está baseado em astronomia babilônica 3]. 
observações com o objetivo de usá-los em astrologia. A esse respeito os historiadores comumente aceitam que eles usaram estas observações para determinar a posição dos corpos celestes, mas não usaram estas observações como base para construir teorias sobre o Universo. Isto fica evidente na compreensão padrão do conhecimento babilônio expressado por Koyre:

[...]por certo, podemo-nos perguntar se não devemos recuar mais longe no tempo e se não devemos colocar a origem da astronomia e da cosmologia científica, não na Grécia, mas na Babilônia. Parece-me haver duas razões para não fazê-lo. Uma está ligada ao fato de que os babilônios nunca se desembaraçaram da astrobiologia que Masson-Oursel acaba de evocar e de que a Grécia consiguiu fazê-lo (aliás, é possível que, na Grécia, a astrobiologia não tenha sido absolutamente um fenômeno original, mas pelo contrário, um fenômeno tardio, muito posterior à origem da astronomia). A outra razão é menos histórica: está ligada à própria noção que atríbuimos à ciência e do trabalho científico. Com efeito, se admitíssemos uma certa concepção ultrapositivista e ultrapragmática da ciência e do trabalho científico, certamente deveríamos dizer que foram os babilônios que começaram. Realmente, eles observaram os céus, fixaram as posições das estrelas e organizaram os respectivos catálogos, anotando, dia a dia, as posições dos planetas. Se isso é feito cuidadosamente durante séculos, chega-se, no fim das contas, a ter catálogos que revelarão a periodicidade dos movimentos planetários e oferecerão a possibilidade de prever, para cada dia do ano, a posição das estrelas e dos planetas que serão reencontrados cada vez que se olhar para o céu. O que é muito importante para os babilônios, pois, dessa previsão das posições dos planeta, depende, pelos caminhos da astrologia, uma previsão dos acontecimentos que se darão na Terra. Assim, se a previsão e a predição equivalem a ciência, nada é mais científico do que a astronomia babilônica. Mas se vir no trabalho científico, sobretudo, um trabalho teórico e se se acreditar - como é meu caso - que não há ciência onde não há teoria, rejeitar-se-à a ciência babilônica e dir-se-á que a cosmologia científica dá seus primeiros passos na Grécia, pois foram os gregos que, pela primeira vez, conceberam e formularam a exigência intelectual do saber teórico: preservar os fenômenos, isto é, formular uma teoria explicativa do dado observável, algo que os babilônios jamais fizeram [4, p.84-85].

Este pensamento histórico padrão sobre os babilônicos, apresentado de forma bastante sustentável por muitos historiadores da ciência, está atualmente sendo questionado, recentemente Mathieu Ossendrijver ${ }^{3}$ publicou um artigo onde apresenta uma reanálise de uma tábua cuneiforme onde está registrado observações do planeta Júpiter entre os séculos IV e II a.C., mostrando evidências que os antigos babilônios não só usavam cálculos aritméticos para determinar a posição do planeta, mas também cálculos geométricos similares ao cálculo do método "área sob uma curva"combinando conceitos como tempo, distância e velocidade, este método foi registrado historicamente no século XIV pelo grupo dos calculadores de Oxford [5].

Todas estas questões trazem reflexões sobre as fontes históricas e sobre a evolução dos conceitos para realizar a reconstrução do conhecimento astronômico antigo, e de um dado modelo astronômico em uma dada época da história. É interessante observar que os conceitos atualmente considerados como padrão podem e devem ser repensados a cada nova descoberta histórica. Com estas considerações neste artigo vamos apresentar em forma detalhada o primeiro modelo matemático da história da astronomia, atualmente aceito, isto é, o modelo das esferas concêntricas de Eudoxo, mas sem deixar de mencionar as limitações de fontes escritas para sua reconstrução.

Começamos apresentando um panorama geral da astronomia grega nos séculos IV e V a. C. Logo se apresentam as ideias cosmológicas de Platão que influenciaram o modelo de Eudoxo, depois, analisamos as duas principais fontes escritas do modelo de Eudoxo: os comentários de Aristóteles e de Simplício. Na seguinte seção apresentamos a reconstrução matemática do modelo de Eudoxo proposta pelos historiadores do século XIX, o chamado século da história, nesta seção seguimos a reconstrução mostrada por Neugebauer [2] e [1], sendo que ele mesmo faz contribuições originais. Na seguinte seção discutimos uma reconstrução moderna do modelo de Eudoxo usando o método das matrizes de rotação, desta forma podemos escrever a equação da hipópede em forma paramétrica e numa linguagem moderna. Logo discutimos as críticas à reconstrução histórica do século XIX e recentes questões publicadas sobre o modelo de Eudoxo. Neste trabalho nos restringimos matematicamente ao "modelo original de Eudoxo" aplicado aos planetas, sendo que unicamente comentamos as modificações feitas por Cálipo e Aristóteles 6]. ${ }^{4}$

\section{Astronomia grega dos séculos V e IV a.C. e a influência de Platão no modelo de Eudoxo}

Em torno dos séculos V e IV a.C. os gregos tinham importantes conhecimentos astronômicos, por exemplo, conheciam que o Sol se deslocava seguindo uma faixa conhecida como eclíptica. Neste sentido, o movimento do

\footnotetext{
${ }^{3}$ Para olhar artigos e textos sobre recentes progressos da astronomia babilônica acessar: https://amor.cms.hu-berlin.de/ ossendrm/ cuneiform.html

${ }^{4}$ As modificações feitas por Cálipo também podem ser modeladas matematicamente, ver a respeito a referência 6], no entanto, as modificações de Aristóteles não tem uma modelagem matemática porque são carentes de lógica.
} 
Sol era descrito ao longo do ano por uma faixa chamada de zodíaco, onde uma das principais preocupações dos gregos aqui, foi a de desenvolver um calendário usando o movimento da Lua e do Sol, o chamado ciclo de Menom (475 - 431 a.C.) proposto por Menom de Atenas 7].

Este conhecimento sobre o Sol foi em grande parte construído pelo uso do gnômon que segundo Heródoto (485 - 420 a.C.) foi inventado pelos babilônios e introduzido na Grécia por Anaximandro de Mileto (610 - 546 a.C.), o qual consiste de uma haste colocado em forma vertical sobre uma superfície plana, e de modo simples, a posição do Sol no céu era identificada pela sombra da haste. Com este aparelho os gregos contavam os dias entre um solstício e um equinócio observando que as estações não têm o mesmo número de dias, isto aparentemente foi feito por Euctêmon (? - 432 a.C.), contemporâneo de Sócrates (469 - 399 a.C.). Este fato observacional permitiu inferir que o Sol não deveria ter uma velocidade constante ao longo da eclíptica, no entanto, isso resultou contrário as ideias metafísicas de Platão (427 - 347 a.C.), onde os corpos celestes tinham que ter movimentos perfeitos e velocidades constantes, como também trajetórias circulares 8.

Os gregos também sabiam distinguir e usar as diferentes fases da Lua, inclusive menciona-se algumas predições de eclipses solares, como a famosa predição feita por Tales de Mileto (624 - 526 a.C.), contada pelo historiador Heródoto, que permitiu assinar um acordo de paz entre Medos e Lídios, ou a famosa predição do eclipse solar de Hélicon, contado por Plutarco (45 - 127 d.C.). Sobre a predição de Tales pode ser simplesmente uma lenda, pois, não há fontes documentais e precisas sobre esta predição. Mas, por exemplo, na referência [9] são discutidas algumas evidências em favor da possibilidade de um erudito, da época de Tales, ter as ferramentas e condições necessárias para poder realizar a mencionada predição.

Outro fato interessante, é o movimento diário das estrelas do leste a oeste, voltando todos os dias às mesmas posições. Adicionalmente, outra observação de grande importância se refere ao movimento dos planetas, que vistos pelos antigos eram estrelas que pareciam ter movimentos contrários em algumas partes de seu percurso ao longo de um dado período. O movimento dos planetas, cujo termo etimologicamente significa "errantes", foi fundamental para o desenvolvimento da astronomia e da matemática. Por exemplo, Platão no Timeu, um livro de forte inspiração pitagórica, ao respeito menciona,

A partir do raciocínio e do desígnio de um deus em relação à geração do tempo, para que ele fosse engendrado, gerou o Sol, a Lua e cinco astros, que têm o nome "planetas", para definirem e guardarem os números do tempo. Tendo construído os corpos de cada um deles - sete ao todo -, o deus estabeleceu-os nas órbitas que o percurso do Outro seguia, em número de sete delas: na primeira a Lua, à volta da Terra; na segunda o Sol, por cima da Terra; a Estrela da Manhã e o astro que di- zem ser consagrado a Hermes na rota circular que tem a mesma velocidade que o Sol, ainda que lhes tenha cabido em sorte um ímpeto contrário ao dele. Daí decorre que o Sol e a Estrela da Manhã (o astro de Hermes) sucessivamente se alcancem e sejam alcançados mutuamente. [8, p.111]

Este parágrafo do Timeu apresenta de forma resumida a cosmologia platônica, e menciona explicitamente que Mércurio (Hermes) e Vênus (Estrela da manhã) se movimentam próximos do Sol (menciona também que ambos têm a mesma velocidade), e que em algum momento eles têm um ímpeto contrário, que pode ser interpretado como um movimento retrógrado. Desta forma, podemos considerar que Platão tinha consciência do movimento retrógrado dos planetas.

Por outro lado, os pitagóricos ensinavam que a Terra era uma esfera, e que cada um dos sete objetos celestes: o Sol a Lua e os cinco planetas tinham uma esfera, e que existia uma distância harmônica entre cada esfera e a Terra. Surge então a ideia da harmônia das esferas [10], a qual entra na história do pensamento em diversas oportunidades, sendo uma das mais importantes a tentativa de Kepler (1571 - 1630) de ajustar as distâncias dos planetas a esta suposta harmonia 5 Desta forma podemos observar que a ideia do movimento dos astros sobre uma esfera, assim como, a trajetória circular, ficou estabelecido entre os filósofos e astrônomos do século IV a.C. A esfera das estrelas fixas, com seu movimento repetitivo todas as noites, representa uma imagem da perfeição intimamente ligada às ideias platônicas.

Dentro deste contexto, Eudoxo de Cnido (408 - 355 a.C.), propõe seu modelo astronômico ou cosmológico, ${ }^{6} \mathrm{o}$ qual é considerado o primeiro modelo matemático (geométrico) na história da ciência para explicar os movimentos dos objetos celestes. Com este modelo se deslocam os estudos da pura especulação filosófica, dos pré-socráticos, para a construção de modelos geométricos, que irão se constituir no método aplicado pelos subsequentes astrônomos gregos. No entanto, os astrônomos da antiga Grécia substituíram o modelo de Eudoxo pelo modelo dos epiciclos, de Ptolomeu, isto foi feito, em grande parte, pelo fato da procura por um modelo matemático de maior precisão para fazer predições e comparações com as observações.

Tudo o que sabemos sobre Eudoxo é de forma indireta, por exemplo, do texto de Diógenes Laércio (180 240 d.C.) sabemos que Eudoxo deve ter frequentado à academia de Platão, em Atenas, em torno do ano 380 a.C., e como estudante deve ter se familiarizado com as ideias cosmológicas de Platão. Também o mesmo Laércio afirma que Eudoxo teve como mestre de matemática a

\footnotetext{
${ }^{5}$ Para uma revisão detalhada sobre a harmonia das esferas ao longo da história ver a referência menciona 10

${ }^{6}$ Atualmente é importante diferenciar a astronomia da cosmologia, mas aqui, mencionamos modelo cosmológico como sinônimo de modelo astronômico, no sentido que o modelo das esferas concêntricas representa um modelo para todos os objetos celestes conhecidos da época e, portanto, um modelo para todo o Universo.
} 
um pitagórico, Arquitas de Tarento (428 - 347 a.C.), o que pode explicar sua proximidade com a ideia da esfericidade da Terra e dos movimentos celestes. Também se sabe que visitou o Egito, em particular a cidade de Heliópolis, que na época era um dos principais centros de conhecimentos astronômicos. Sabe-se ainda que voltou a Atenas e teve discordância com Platão, mas outros relatos mostram que Platão o tinha em alta estima pelos seus grandes conhecimentos matemáticos. Eudoxo era um dos poucos que sabia a solução para o problema da duplicação do cubd ${ }^{7}$

Eudoxo como um astrônomo de seu tempo ficou fortemente influênciado por Platão, o qual não era somente um respeitado erudito, mas também tinha um coletivo de pensadores que frequentavam sua Academia, os quais espalhavam suas principais ideias. Simplício menciona que foi Platão quem propôs a questão de buscar um modelo cosmológico para "Salvar os fenômenos". Alguns historiadores da ciência como Koyré estabeleceram que esta frase significa explicar com uma teoria o movimento dos objetos celestes, usando o círculo como trajetória perfeita para os astros, sem inicio e fim, e também a ideia da velocidade constante. Atualmente temos poucas formas de provar que a frase mencionada por Simplício seja de fato de Platão. Neste caso, vale lembrar que Simplício escreveu no século VI d.C. Portanto, depois de muitos séculos do modelo de Eudoxo ter sido proposto, mesmo se levarmos em conta que Simplício pode ter lido textos atualmente desconhecidos, esperariamos que citasse suas fontes, como ele fez em outros casos, também poderia ter sido um conhecimento comum na época de Simplício, seja como for, a questão de "Salvar os fenômenos"é compatível com a cosmologia de Platão no Timeu [8]

Os comentadores antigos mencionam que Eudoxo escreveu dois livros associados com astronomia: Espelhos e Fenômenos. No entanto, mesmo não tendo os livros é possível conhecer indiretamente o conteúdo do livro Fenômenos, devido ao poeta Arato (315 - 240 a.C.) que escreveu um poema denominado "Fenômenos"usando como fonte de inspiração o texto de Eudoxo. Neste texto, entre outras coisas, se apresentam informações sobre as constelações e o uso da esfera para descrever o movimento dos objetos celestes. O poema de Arato foi muito reproduzido na sua época, sendo que o grande astrônomo Hiparco (190 - 120 a.C.) menciona tê-lo utilizado.

As duas principais fontes escritas para conhecer o modelo astronômico de Eudoxo são os escritos de Aristóteles e de Simplício. Aristóteles pode ser considerado uma geração mais jovem que Eudoxo e, portanto, uma fonte contemporânea. Por outro lado, Simplício, como mencionado, foi um comentador do século VI d.C., isto é, quase 900 anos após o modelo de Eudoxo. Simplício fundamentou seus comentários nos escritos de Sosígenes (Séc II a.C.), que por sua vez, usou o livro "Historia da Astrono-

\footnotetext{
${ }^{7}$ A duplicação do cubo ou problema de Delos, é um dos três problemas famosos da antiguidade, é um problema de geometria que consiste na construção de um cubo com régua e compasso, com o dobro do volume de um cubo conhecido, utilizando apenas uma aresta deste.
}

mia", escrito por Eudemo de Rodes (370 - 300 a.C.), que foi estudante na escola peripatética de Aristóteles, escrevendo vários livros sobre a história das ciências gregas. Além de Simplício há registros do trabalho de Eudemo em escritos de Téon de Esmirna (70 - 135 d.C.), Diógenes Laércio e Clemente de Alexandria (150 - 215 d.C.).

\section{Modelo de Eudoxo nos escritos de Aristóteles e Simplício}

Os argumentos de Aristóteles sobre o modelo de Eudoxo são apresentados em seus tratados de metafísica, especificamente, no livro XII ou $\Lambda^{8}$ este texto começa com uma apresentação sobre a questão do primeiro motor do movimento celeste, o qual é definido como uma entidade eterna e imóvel. Depois se considera o número dos objetos em movimento, para o qual ele menciona que esta questão deve ser considerada a partir do ponto de vista do conhecimento astronômico. Aristóteles pondera que os estudos da matemática devem incluir além da aritmética e da geometria a astronomia. Depois destas considerações passa a mencionar a teoria das esferas concêntricas, e as modificações de Cálipo de Cízico (370 - 300 a.C.), que foi discípulo de Eudoxo, e depois ele passa a fazer suas próprias modificações. Nesse mesmo texto Aristóteles apresenta como Eudoxo definiu as esferas concêntricas:

Eudoxo supõe que o movimento do Sol ou da Lua envolve, em ambos os casos, três esferas, das quais a primeira é a esfera das estrelas fixas, e a segunda se move no círculo ao longo do meio do zodíaco, e a terceira no círculo que está inclinado com respeito ao zodíaco; mas o círculo em que a Lua se move está inclinado em um ângulo maior do que aquele em que o Sol se move. E o movimento dos planetas envolve, em cada caso, quatro esferas, e que as primeiras e as segundas são as mesmas que as duas primeiras acima mencionadas ... os polos da terceira esfera de cada planeta estão no círculo do zodíaco, e o movimento da quarta esfera está no círculo inclinado em um ângulo do equador da terceira esfera, e os polos da terceira esfera são diferentes para cada um dos planetas, mas para Vénus e Mercúrio são os mesmos. 11, Livro XII, tradução nossa]

Na explicação sobre o movimento dos planetas fica claro a definição das quatro esferas, sendo que as duas primeiras são usadas de forma similar às esferas do Sol e da Lua para explicar seus movimentos diários (primeira esfera) e anuais (segunda esfera). Mas o uso das outras duas esfera não são explicitamente mencionadas nesta descrição, e ainda não menciona o movimento retrógrado. Outra questão interessante, é a afirmação sobre os planetas Mércurio e Vênus, os quais têm em comum o eixo

${ }^{8}$ Os livros que formam o tratado da Metafísica de Aristóteles são etiquetados mediante letras gregas, por isso, o livro XII é conhecido como livro $\Lambda$. 
da terceira esfera, o que pode ser compreendido através das observações destes planetas, pois, seus movimentos aparentes vistos a partir da Terra não se afasta muito da trajetória do Sol ao longo da eclíptica.

Aristóteles ao mencionar as correções feitas por Cálipo de Cízico, menciona que este adicionou duas esferas a mais para o Sol e para a Lua, e para os planetas adicionou uma esfera a mais, salvo para Júpiter e Saturno, que manteveram as mesmas quatro esferas do modelo original de Eudoxo. Aristóteles não detalha como seriam colocadas geometricamente estas outras esferas, em conjunto com as anteriores, e tampouco para que seriam usadas.

Suas próprias modificações parecem ter sido guiadas pela ideia da realidade física das esferas. Para Aristóteles estas esferas eram reais. Mas se questionar sobre a realidade das esferas implica uma série de questões. Por exemplo, que mecanismos físicos mantém os planetas sobre as esferas? Ou como os planetas trocam de esferas? Ou como o movimento é transmitido entre as esferas? Entre outras. Todas estas perguntas ficam sem uma resposta concreta e as modificações de Aristóteles resultam um tanto confusas, e não permitem formular um modelo

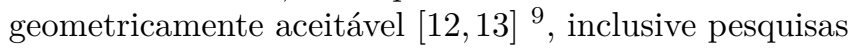
recentes de história da ciência mencionam que Aristóteles em diversos escritos sobre astronomia era suscetível a expressar comentários incoerentes [14, 15].

A segunda fonte histórica escrita sobre o modelo de Eudoxo são, os comentários de Simplício sobre Aristóteles, os quais comparados com o texto da Metafísica são mais precisos e extensos. Sobre a terceira e quarta esferas dos planetas Simplício menciona o seguinte:

A terceira esfera, que tem seus polos no grande círculo da segunda esfera, que passa pelo meio dos signos do zodíaco, e que gira do sul ao norte e do norte ao sul, se movimenta também com a quarta esfera, sendo que o planeta também está ligado à quarta esfera. Além disso, será a causa do movimento do planeta na latitude. Se consideramos o movimento do planeta unicamente sobre a terceira esfera, ele poderia chegar aos polos do círculo do zodíaco, e se aproximaria dos polos do universo; no entanto, como são as coisas, a quarta esfera gira sobre os polos do círculo inclinado, com respeita à terceira esfera, no sentido oposto, isto é, de leste para o oeste, mas no mesmo período, o que impedirá qualquer divergência considerável (por parte do planeta) sobre o círculo do zodíaco e fará com que o planeta descreva sobre este mesmo círculo, do zodíaco, a curva chamada por Eudoxo de hipópede; de modo que, a amplitude desta curva será a quantidade (máxima) do desvio aparente do planeta na latitude, uma

\footnotetext{
9 também revisar o livro de J.L.E Dreyer 7 . History of the planetary Systems from Thales to Kepler para uma explicação detalhada.
}

visão pela qual Eudoxo foi atacado. 16, p.202, tradução nossa].

Nesta citação observar-se a explicação do movimento contrário entre a terceira e quarta esferas, também é mencionada a figura que se forma: a hipópede, a qual se associa com o movimento retrógrado. No entanto, como mencionado, Simplício escreveu no século VI d.C. e mesmo nessa época ele não baseia seus comentários sobre os escritos originais de Eudoxo, mas sobre os escritos de Eudemo e Sosígenes. Outra questão interessante dos textos de Simplício se refere ao questionamento sobre a regularidade das esferas concêntricas. Neste modelo os astros apresentam movimentos uniformes sobre as esferas, com distâncias fixas a partir da Terra, o que notadamente não era verdade, pois as estrelas observadas desde a Terra, de tempos em tempos, variam sua intensidade, o que já era associado à distância por alguns filósofos, segundo ele:

[...] quero dizer é que as vezes os planetas parecem ficar perto, e outras em que parecem ficar longe. E no caso de alguns isso é aparente a simples vista. Pois a estrela que chamamos Vênus e também a que chamamos Marte parecem muitas vezes maiores quando estão na metade de suas retrogradações, de forma que nas noites sem Lua, Vênus provoca que os corpos projetem sombras. [16, p. 221, tradução nossa].

É interessante notar que Simplício considerava que todos os planetas tinham luz própria, pois como comenta Weinberg [12], se considerarmos o Sol como fonte luminosa e os planetas como refletindo a luz Solar, mesmo no modelo de Eudoxo, os planetas teriam mudanças de brilho como consequência de suas diferentes posições relativas entre a Terra e o Sol, mas mesmo assim, não explicariam as mudanças observadas.

\section{Reconstrução do Modelo de Eudoxo}

Uma reconstrução matemática do modelo de Eudoxo fundamentada nas fontes históricas, anteriormente discutidas, foi desenvolvida no início do século XIX, começando com as discussões apresentadas nos trabalhos do alemão Schaubach (1802) 17], de Ideler (1828) [18, e Apelt [19], sendo que o mecanismo matemático completo foi especificado por Schiaparelli (1874) [20]. Também no século XX, Neugebauer(1953) [2] fez importantes contribuições, com discussões originais sobre a reconstrução matemática das esferas concêntricas. O caráter histórico desta reconstrução de Neugebauer se nota a partir do momento em que este busca o contexto matemático da época de Eudoxo. Com base nas suas ilustrações e descrições dos movimentos planetários, exporemos de forma detalhada, o desenvolvimento geométrico para representar o movimento aparentes dos cinco planetas conhecidos, a partir das duas esferas mais internas, pois o movimento do Sol e 
da Lua e das estrelas fixas não demandam um mecanismo adicional ao simples mecanismo das esferas concêntricas. Por outro lado, os cinco planetas conhecidos na época, apresentavam o movimento retrógrado o que faz com que o caráter geométrico tenha que ser mais sútil.

Com base nas Figuras 1] e 2, onde se apresentam a terceira e a quarta esfera para um dado planeta, podemos observar que os eixos principais das duas esferas formam um ângulo $\alpha$, e o planeta se encontra inicialmente no ponto $A$ que representa a interseção entre os equadores das duas esferas. A eclíptica está representada pelo equador da terceira esfera. No modelo de Eudoxo o movimento do planeta está restringido à faixa formada entre os dois equadores, que por sua vez, depende do ângulo de inclinação. Na reconstrução do século XIX se levou em conta o conhecimento de que os planetas em seu movimento aparente ao longo do ano, vistos a partir da Terra, sempre permanecem próximos da eclíptica, portanto, a faixa para todos os planetas é estreita, ou dito de outra forma, as variações em latitude e longitude são pequenas. Em seguida, ao se completar a circunferência, retornando ao ponto $A$, se forma uma Figura que os gregos denominaram de hipóped $\AA^{10}$

Para apresentar a reconstrução geométrica vamos a usar a Figura 2, onde se representa um quarto do movimento planetário, ou mais específico, o quadrante entre as retrogradações. Através das rotações iguais e contrárias e da inclinação entre as esferas, pode-se levantar os seguintes argumentos geométricos [2]:

- Primeiro, o planeta do ponto $A$ se movimenta na quarta esfera, no sentido horário, até o ponto $P_{1}$,

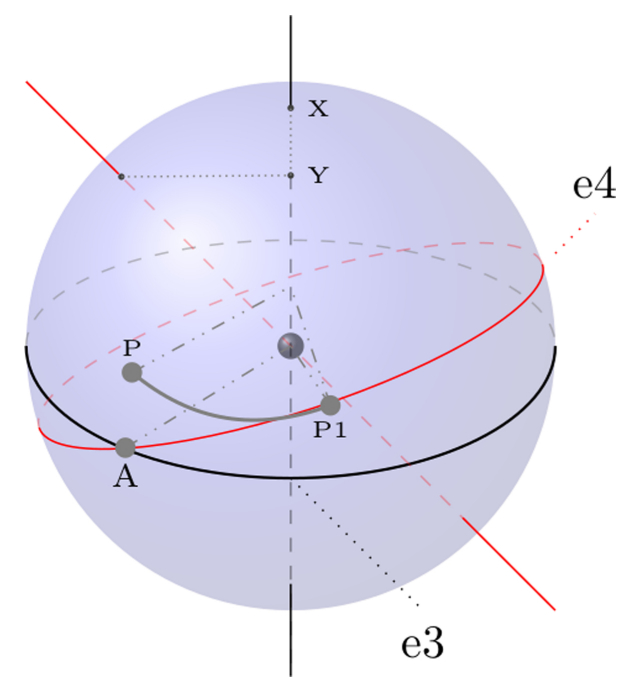

Figura 1: Representação do movimento do planetário. Começando em A o planeta se move no equador vermelho e depois de transfere para o equador em preto até o ponto $P$.

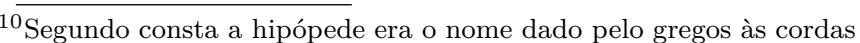
usadas para amarrar cavalos que tinham o formato de oito. Seu uso é antigo, por exemplo, se menciona nos comentários do primeiro livro de Euclides, escrito por Proclo (410-485 d.C.) 7].
}

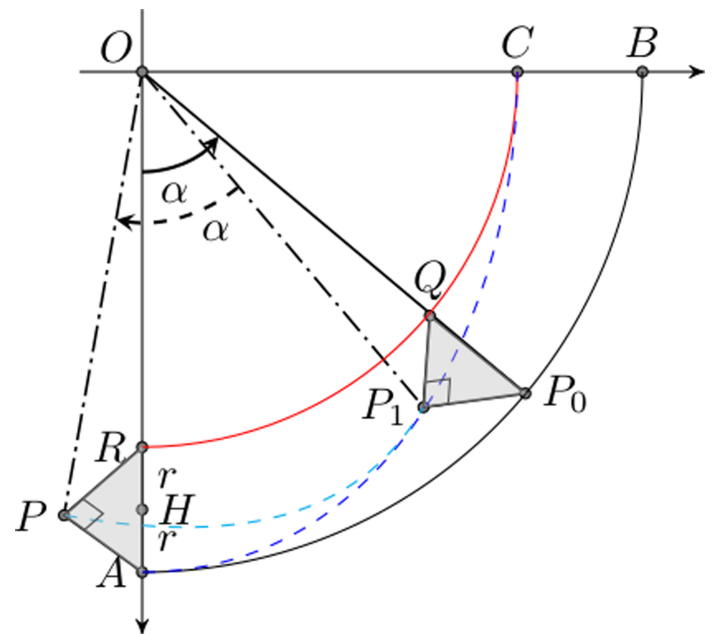

Figura 2: Projeção da quarta esfera sobre o equador da terceira esfera, construida com base na referência [2].

depois ao longo da terceira esfera, no sentido antihorário, até o ponto $P$;

- Se olharmos perpendicularmente para baixo, a partir do polo superior da Figura 1 podemos construir a projeção do movimento entre $A$ e $P$, como ilustra a Figura 2, onde as curvas $\overparen{A B}$ e $\overparen{A C}$ representam $1 / 4$ de circunferência. $O$ planeta segue na trajetória inclinada até o ponto $P_{1}$, mas se ignorarmos a inclinação seguiria até $P_{0}$.

- Com essas considerações podemos construir um segmento de reta $\overline{P_{1} P_{0}}$ perpendicular ao raio $\overline{O A}$ e paralelo a $\overline{P A}$. Se $\overline{C B}=\overline{Q P_{0}}=\overline{A R}$ e $\overline{P_{1} Q} / / \overline{O A}$, construímos dois triângulos retângulos congruentes $\triangle P_{0} Q P_{1}$ e $\triangle A R P$ de tal forma que seus ângulos em $\angle Q$ e $\angle R$ sejam iguais a $\alpha$, pois são ângulos correspondentes.

- Esta construção geométrica nos garante a simetria nos movimentos de rotação entre as esferas, e a formação da base do cilindro de raio $r$ e diâmetro $\overline{A R}$.

Eudoxo foi o primeiro a ir além do mero raciocínio filosófico sobre a construção do universo, e o primeiro a tentar descrever o movimento planetário por meio de um simples raciocínio geométrico, a descrição geométrica anterior resulta em concordância com os desenvolvimentos geométricos da época, comum aos filósofos gregos, diferente das demonstrações de Schaubach ou Schiaparelli, que apresentam soluções trigonométricas complexas que ainda não eram conhecidas na época de Eudoxo. No que segue vamos dar detalhes geométricos do modelo para leitores interessados em aprofundar o desenvolvimento geométrico, no entanto, para vizualizar graficamente como funciona o modelo, o leitor pode fazer uso das diferentes 
ferramentas encontradas na internet ${ }^{11}$ que facilitam a compreensão geral do modelo.

\subsection{Movimento dos planetas nos laços da hipópede}

Ao observarmos as Figuras 1] e 2, nota-se que o que determina o movimento dos planetas entre as esferas é o ângulo $\alpha$, que pode ser considerado como um grau de liberdade que caracteriza o sistema. Seu valor vária de acordo com o astro que está sendo considerado. Com base no que apresenta Neugebauer [1], utilizando os argumentos geométricos anteriores, é possível encontrar a velocidade do planeta ao percorrer os laços da hipópede. Se associarmos o ângulo $\alpha$ da Figura 2 ao ângulo $\gamma$ da Figura 3 e considerarmos que qualquer modificação nesses ângulos produzirá uma variação no segmento $\overline{X Y}$, e ainda que $\overline{X Y}=\overline{B C}$ ou pela Figura 2 que $\overline{B C}=2 r$, podemos facilmente identificar que o grau de liberdade $\alpha$ pode ser representado também pelo raio $r$ do cilindro. Assim, tendo em vista o raio do cilindro, tanto nos triângulos da Figura 2 , quanto da Figura 3 , e a partir do triângulo $\triangle R O S$ e o arco de seguimento $\overline{O A}=1$ unitário, podemos escrever,

$$
\cos \gamma=\frac{\overline{O R}}{O S}
$$

Como $\overline{O S}=\overline{O A}=1$ fica,

$$
\cos \gamma=\overline{O R},
$$

Ainda da Figura 3 vemos que,

$$
\overline{O A}=\overline{O R}+\overline{R A} \text {. }
$$

E sendo $\overline{R A}=2 r$, o raio $r$ será dado por,

$$
\begin{aligned}
& 1=\cos \gamma+2 r \\
& r=\frac{1}{2}(1-\cos \gamma)
\end{aligned}
$$

Portanto, fica assim estabelecido a dependência de $r$ diretamente do ângulo entre os eixos das esferas. Essa equivalência nos garante a formação e o tamanho do cilindro, ou pelo menos de sua base como mostra a Figura 3 .

Agora vamos a desenvolver mais detalhadamente o modelo de Eudoxo determinando a longitude ${ }^{12}$ que na Figura 3 é representada pelo ângulo $\beta$, o qual subtende o arco $\overparen{P P_{e}}$. Para uma melhor visualização a Figura 4 apresenta os detalhes, e se observa que devido ao triangulo $O P P^{\prime \prime}$ é possível definir,

\footnotetext{
${ }^{11}$ Por exemplo: https://www.youtube.com/watch?v=_SFzDYSqR_4 onde se apresenta de forma gráfica o modelo de Eudoxo passo a passo. Nos videos: https://www youtube. $\operatorname{com} /$ watch? $\mathrm{v}=$ hhikvgDVcGY e https://www. youtube.com/watch?v=I4o0u8wINas se apresentam uma comparação gráfica entre os modelos de Eudoxo, Ptolomeu e Copérnico.

${ }^{12}$ Resulta importante mencionar que este desenvolvimento que vamos a apresentar, usando funções trigonometricas, não era conhecido na época de Eudoxo.
}

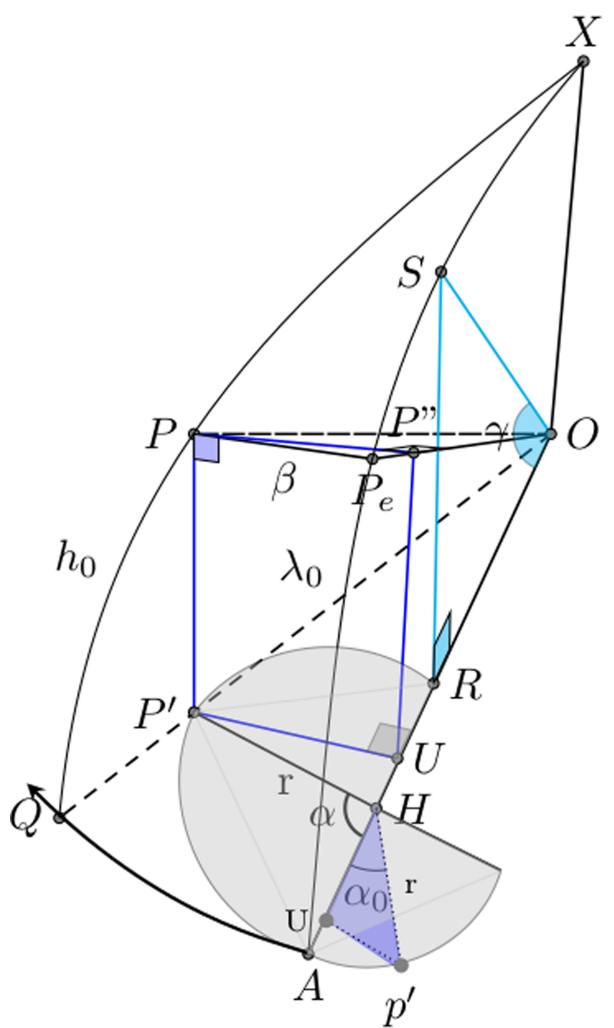

Figura 3: Construção geométrica fundamentada na construção proposta por Neugebauer para determinar a velocidade do planeta. Interessante notar que a hipópede nesta construção tem seus laços na vertical, portanto, quando falamos de longitude falamos de segmentos de curvas na vertical.

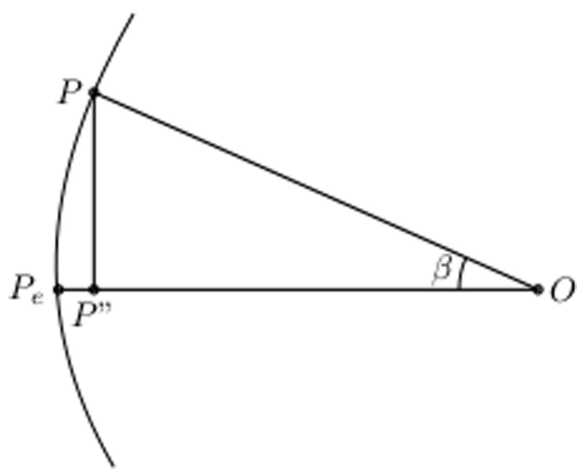

Figura 4: Triângulo mostrando o ângulo $\beta$ baseada na geometria mostrada na Figura 3

$$
\sin \beta=\frac{\overline{P P^{\prime \prime}}}{\overline{O P}}
$$

onde $\overline{O P}=1$ assim obtemos

$$
\sin \beta=\overline{P P^{\prime \prime}}=\overline{P^{\prime} U}
$$

Onde levamos em conta a construção geométrica da Figura 3 que mostra que o segmento $\overline{P P^{\prime \prime}}=\overline{P^{\prime} U}$. De forma similar escrevemos a função cosseno, 


$$
\cos \beta=\frac{\overline{O P^{\prime \prime}}}{\overline{O P}} \rightarrow \overline{O P^{\prime \prime}}=\overline{O U}
$$

A tangente fornecerá $\beta_{\max }$, mas só existe o valor máximo se $\overline{P^{\prime} U}=r$ e $\alpha=90$. Assim usando as equações (4) e (7) obtemos,

$$
\tan \beta=\frac{\sin \beta}{\cos \beta}=\frac{\overline{P^{\prime} U}}{\overline{O U}} .
$$

Mas usando o fato que $\overline{O U}=\overline{O A}-\overline{U A}=1-r$ quando $\alpha=90$ (o ponto $U$ é uma projeção que percorre o diâmetro $\overline{A R}$ do cilindro na base da Figura 3 , a equação (8) se reescreve,

$$
\tan \beta=\frac{r}{1-r}
$$
fica,

Substituindo a equação (4) na equação (9) a tangente

$$
\tan \beta=\frac{1-\cos \gamma}{1+\cos \gamma}
$$

Com este resultado se pode determinar a velocidade do ponto $P$ no circulo de diâmetro $\overline{A R}$, isto pode ser obtido analisando a projeção de $P$ na base da Figura 3 ou seja o ponto $P^{\prime}$. A componente longitudinal do movimento de $P$ atinge velocidade máxima $v_{0}$ em $A$. Podemos estimar o valor $v_{0}$ diretamente desta projeção para um curto intervalo de tempo $t_{0}$, durante o qual a projeção $P^{\prime}$ de $P$ se move na base do cilindro através de um ângulo $\alpha_{0}$ detalhado na Figura 5 partindo de $A$ até o ponto $p^{\prime}$, e se considerarmos que a velocidade angular de $P^{\prime}$ é o dobro da velocidade de cada uma das esferas, temos;

$$
\frac{t_{0}}{\Delta t}=\frac{\alpha_{0}}{2 \cdot 360}
$$

onde $\Delta t$ é o período sinódico. Agora se $\lambda_{0}$ é o segmento de arco $\widehat{A P_{e}}$ o qual consideramos uma pequena variação longitudinal para poder fazer a seguinte aproximação $\overparen{A P_{e}} \approx \overparen{Q P}$ e portanto $\lambda_{0} \approx h_{0}$. Definimos a velocidade em função destes parâmetros estabelecidos, logo:

$$
v_{0}=\frac{\lambda_{0}}{t_{0}} .
$$

Usando a equação 11 a velocidade pode ser escrita como,

$$
v_{0}=\frac{h_{0}}{\alpha_{0}} \cdot \frac{720}{\Delta t}
$$

Para poder ter uma expressão para a velocidade em função do ângulo entre as duas esferas, vamos a calcular $h_{0}$ como função de $\alpha_{0}$. Para fazer isto tracemos um segmento de $P_{e}$ passando por $U$ até $p^{\prime}$, ou seja, a projeção $P^{\prime}$ sai do lado esquerdo passa por $A$ até $p^{\prime}$ de modo que $\alpha_{0}$ seja mínimo possível.

Usando o triângulo da Figura 5 podemos calcular uma relação para o ângulo $h_{0}$ do seguinte modo,

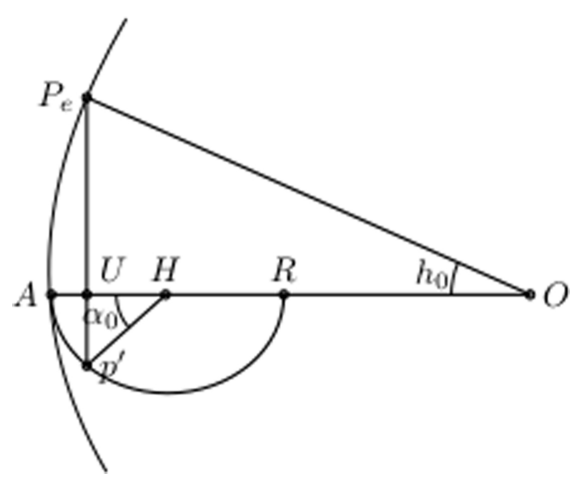

Figura 5: Triângulo mostrando a geometria usada para escrever $h_{0}$ em função de $\alpha_{0}$ baseada na geometria mostrada na Figura 3.

$$
\cosh _{0}=\frac{\overline{O U}}{\overline{O P_{e}}}
$$

E da geometria da Figura 5 podemos obter,

$$
\overline{O U}=\overline{O A}-\overline{U A}=1-\overline{U A}
$$

Logo definimos o segmento $\overline{U A}$ e o $\triangle U H p^{\prime}$ com base nas Figuras 3 e 5 e obtemos as equações (16) e (17) que segue,

$$
\begin{gathered}
\cos \alpha_{0}=\frac{\overline{H U}}{r} \\
\overline{U A}=\overline{H A}-\overline{H U}=r-r \cos \alpha_{0}
\end{gathered}
$$

Com esta relação calculamos a função $\cos h_{0}$ da seguinte forma,

$$
\cos h_{0}=1-r+r \cos \alpha_{0}
$$

Para ângulos muito pequenos $\cos h_{0}$ e $\cos \alpha_{0}$ podem ser decompostos em série de potência até segunda ordem, ficando;

$$
\begin{aligned}
\cosh _{0} & =1-r+r \cos \alpha_{0} \\
1-\frac{h_{0}^{2}}{2} & =1-r+r\left(1-\frac{\alpha_{0}^{2}}{2}\right) \\
& =1-r+r-\frac{r}{2} \cdot \alpha_{0}^{2}
\end{aligned}
$$

Colocando $h_{0}$ como função de $\alpha_{0}$, veremos que;

$$
h_{0}=\sqrt{r} \cdot \alpha_{0} .
$$

Substituindo na equação 13 para $v_{0}$, definimos a velocidade que o planeta desenvolve passando duas vezes no ponto $A$ formando o loop completo.

$$
v_{0}=\frac{h_{0}}{\alpha_{0}} \cdot \frac{720}{\Delta t}=\frac{\sqrt{r} \cdot \alpha_{0}}{\alpha_{0}} \cdot \frac{720}{\Delta t} \cdot=\sqrt{r} \cdot \frac{720}{\Delta t}
$$


Portanto, o movimento final do planeta resulta a soma do movimento sideral, devido ao período sinódico, mais as rotações contrárias das duas esferas mais internas, gerando a interseção do cilindro com a esfera, e o valor do raio do cilindro (ângulo $\alpha$ ) depende do planeta analisado. A Figura 6] mostra a ilustração desses resultados devido a construção da hipópede. É interessante destacar que a equação relaciona o raio do cilindro com a velocidade do planeta, isto é, relaciona a velocidade do planeta com o ângulo $\alpha$ entre as duas esferas internas do modelo de Eudoxo.

\section{As esferas de Eudoxo usando Matrizes de Rotação}

Um método alternativo para demonstrar a trajetória dos planetas no modelo de Eudoxo, usando uma linguagem matemática moderna, resulta do uso das matrizes de rotação 21]. Esta abordagem é mais clara matematicamente e permite construir a hipópede na forma de uma equação paramétrica. Antes de mostrar a aplicação deste método matricial para o caso do modelo de Eudoxo vamos lembrar alguns conceitos básicos de um sistema de coordenadas em rotação.

Considere um sistema fixo de coordenadas cartesianas $X Y$, conforme observamos na Figura 7, e então rotacionamos os eixos por um ângulo $\theta$ de modo a encontrar os eixos $X^{\prime} Y^{\prime}$. Temos então dois pares de eixos, e assim, podemos decompor um vetor $\vec{r}$ fazendo projeções ortogonais, escrevendo as componentes do vetor $\vec{r}$ no sistema rotacionado em função de suas componentes do sistema fixo. Considerando a geometria da Figura 7 observa-se que; $x_{r}^{\prime}=\overline{O B}+\overline{B C}$ e $y_{r}^{\prime}=\overline{O F}-\overline{G F}$. Então $\overline{O B}=x_{r} \cos \theta$.

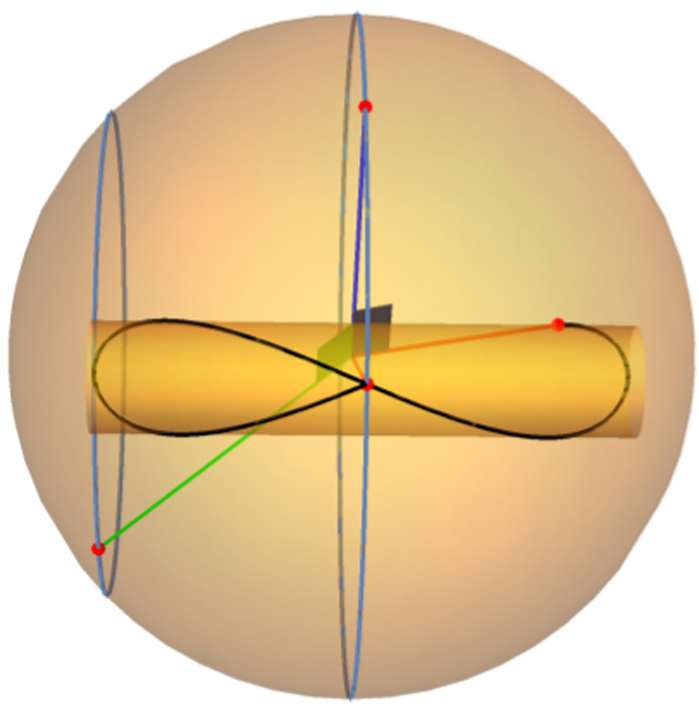

Figura 6: Interseção do cilindro com a esfera. Esta Figura foi feita editando um programa livre feito em Mathematica e extraído do sítio Wolfram Demonstration Project (http: //demonstrations.wolfram.com/HippopedeOfEudoxus/)
Ainda $\overline{B C}=\overline{A D}$, logo obtemos $\overline{A D}=y_{r} \sin \theta$, então $\overline{B C}=y_{r} \sin \theta$, e assim,

$$
x_{r}^{\prime}=x_{r} \cos \theta+y_{r} \sin \theta
$$

De modo semelhante $\overline{O F}=y_{r} \cos \theta$. Os segmentos $\overline{G F}=\overline{E H}$, o que nos permite obter $\overline{E H}=x_{r} \sin \theta$. Sendo que,

$$
y_{r}^{\prime}=y_{r} \cos \theta-x_{r} \sin \theta
$$

Com estas duas equações é possível formar uma matriz para descrever a rotação dos eixos da seguinte maneira;

$$
R(\theta)=\left[\begin{array}{l}
x_{r}^{\prime} \\
y_{r}^{\prime}
\end{array}\right]=\left[\begin{array}{cc}
\cos \theta & \sin \theta \\
-\sin \theta & \cos \theta
\end{array}\right] \cdot\left[\begin{array}{l}
x_{r} \\
y_{r}
\end{array}\right]
$$

Esta matriz representa uma rotação anti-horária de ângulo $\theta$ no plano. Ou seja, $R(\theta)$ é uma transformação que faz uma rotação de $\theta$. Em geral as rotações tem uma estrutura matemática de grupo, o chamado grupo de rotação $S O(3)^{13}$. Por exemplo, se temos uma rotação $R(\theta)$ e outra $R(\alpha)$ a rotação total pode ser representada como $R(\theta)+R(\alpha)=R(\theta) R(\alpha)$.

Para uma correspondência entre o modelo de Eudoxo e os conceitos de matriz de rotação usaremos a Figura 8 Adicionalmente, outras considerações necessárias são as seguintes 21]:

1. Na terceira esfera fixamos um conjunto de vetores unitários $\left(\vec{i}_{3}, \vec{j}_{3}, \vec{k}_{3}\right)$, com $\vec{k}_{3}$ ao longo do eixo de rotação, que por sua vez está no plano da eclíptica e é representada pela esfera externa (conforme Figura 8), formando um ângulo $\alpha$ com $\vec{k}_{1}$ e $\vec{k}_{2}$;

2. A quarta esfera possui uma inclinação $\alpha$ em relação à eclíptica, onde foi introduzido os vetores unitários $\left(\vec{i}_{1}, \vec{j}_{1}, \vec{k}_{1}\right)$, rotacionando ao redor de $\vec{k}_{1}$, que na Figura 8 é representada pela esfera interna.

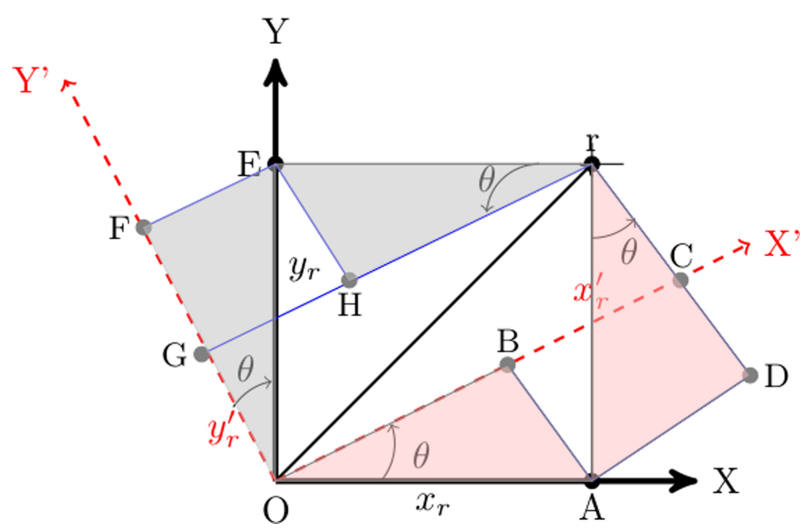

Figura 7: Sistema de Coordenadas usado para produzir uma relação matricial no plano.

\footnotetext{
13 Esse grupo se refere a todas as rotações no espaço tridimensional euclidiano $R^{3}$ sob uma propriedade de composição, preservando a origem, a distância euclidiana e a orientação. Tem muitas aplicações em física teórica, por exemplo em física de partículas.
} 
Observamos ainda que $\vec{i}_{1}$ e $\vec{j}_{1}$ giram no plano equatorial da quarta esfera, e a posição do planeta é representada a todo momento pelo vetor $\vec{i}_{1}$;

3. De modo semelhante consideremos o sistema fixo $\left(\overrightarrow{i_{2}}, \overrightarrow{j_{2}}, \overrightarrow{k_{2}}\right)$, que não rotaciona com nenhuma esfera, mas está relacionado com os vetores $\left(\vec{i}_{1}, \vec{j}_{1}, \vec{k}_{1}\right)$ por meio da matriz de rotação.

4. A terceira e quarta esfera rotacionam em direções opostas uma em relação a outra com mesmo período de revolução.

\subsection{Equação das matrizes de rotação}

Vamos a considerar que a posição do planeta está dada pelo vetor $i_{1}$, o qual esta rotando no plano equatorial da quarta esfera, assim podemos escrever a matriz de rotação entre o sistema ortogonal de vetores da quarta esfera (sistema 1) e o sistema ortogonal de vetores fixo (sistema 2) como,

$$
\left(\begin{array}{l}
\overrightarrow{i_{2}} \\
\overrightarrow{j_{2}} \\
\overrightarrow{k_{2}}
\end{array}\right)=\left(\begin{array}{ccc}
\cos \omega t & -\sin \omega t & 0 \\
\sin \omega t & \cos \omega t & 0 \\
0 & 0 & 1
\end{array}\right) \cdot\left(\begin{array}{l}
\overrightarrow{i_{1}} \\
\overrightarrow{j_{1}} \\
\overrightarrow{k_{1}}
\end{array}\right)
$$

onde consideramos que o sistema da quarta esfera está girando com uma velocidade angular $-\omega$. Agora vamos a determinar a relação entre a rotação da terceira e quarta esferas. Pela simetria do sistema apresentado na Figura 8 podemos decompor o movimento da terceira esfera em dua rotações, uma no eixo de $\overrightarrow{k_{3}}$, e outra no eixo de $\overrightarrow{j_{3}}$, pois podemos afirmar que a inclinação $\alpha$ entre os equadores das esferas é tal que pode ser facilmente identificada entre os vetores unitários $\overrightarrow{j_{3}}$ e $\overrightarrow{j_{2}}$ de cada

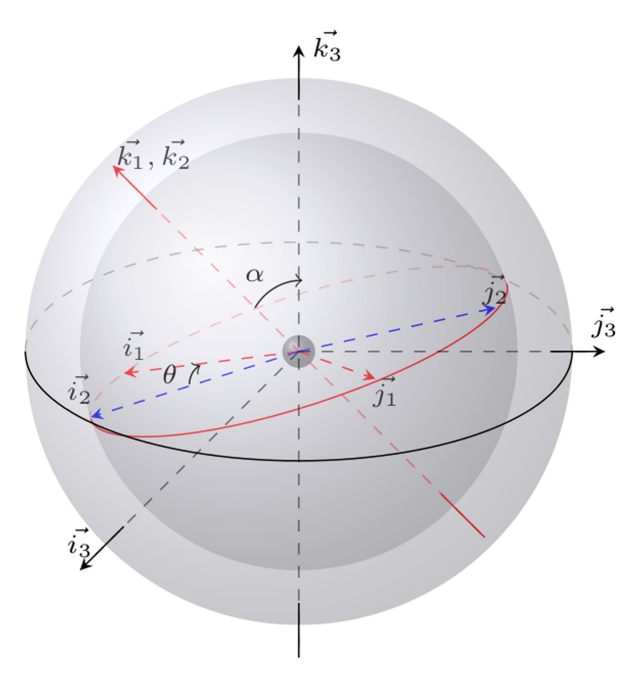

Figura 8: Representação das esferas do movimento retrógrado ao redor da Terra. O planeta esta localizado mediante o vetor unitário $i_{1}$, e na figura é mostrado o sistema móvel 1 girando um ângulo $\theta$ com respeito ao sistema fixo $2 \mathrm{em}$ torno do eixo $k_{1}$ que coincide com $k_{2}$. O eixo da terceira esfera esta situada nos polos da eclíptica. esfera. Assim, podemos escrever a matriz de rotação em um ângulo $\alpha$ ao redor do eixo $j_{3}$ como:

$$
\left(\begin{array}{ccc}
\cos \alpha & 0 & \sin \alpha \\
0 & 1 & 0 \\
-\sin \alpha & 0 & \sin \alpha
\end{array}\right)
$$

Logo utilizando a propriedade de multiplicação de matrizes obtemos o seguinte resultado;

$$
\begin{array}{r}
\left(\begin{array}{ccc}
\cos \omega t & -\sin \omega t & 0 \\
\sin \omega t & \cos \omega t & 0 \\
0 & 0 & 1
\end{array}\right)\left(\begin{array}{ccc}
\cos \alpha & 0 & \sin \alpha \\
0 & 1 & 0 \\
-\sin \alpha & 0 & \sin \alpha
\end{array}\right) \\
=\left(\begin{array}{ccc}
\cos \omega t \cos \alpha & -\sin \omega t & \cos \omega t \sin \alpha \\
\sin \omega t \cos \alpha & \cos \omega t & \sin \omega t \sin \alpha \\
-\sin \alpha & 0 & \cos \alpha
\end{array}\right)
\end{array}
$$

Portanto, o movimento do planeta pode ser escrito da seguinte forma, em função dos vetores unitários $\left(\vec{i}_{3}, \vec{j}_{3}, \vec{k}_{3}\right)$ da terceira esfera;

$\left(\begin{array}{c}\overrightarrow{i_{3}} \\ \overrightarrow{j_{3}} \\ \overrightarrow{k_{3}}\end{array}\right)=\left(\begin{array}{ccc}\cos \omega t \cos \alpha & -\sin \omega t & \cos \omega t \sin \alpha \\ \sin \omega t \cos \alpha & \cos \omega t & \sin \omega t \sin \alpha \\ -\sin \alpha & 0 & \cos \alpha\end{array}\right)\left(\begin{array}{c}\overrightarrow{i_{2}} \\ \overrightarrow{j_{2}} \\ \overrightarrow{k_{2}}\end{array}\right)$.

Podemos determinar a posição do planeta em função do sistema de coordenadas da terceira esfera, $\left(\vec{i}_{3}, \vec{j}_{3}, \vec{k}_{3}\right)$, multiplicando pela matriz $\left(\begin{array}{l}1 \\ 0 \\ 0\end{array}\right)$, pois o planeta está na posição $i_{1}$, assim calculamos,

$$
\begin{gathered}
\left(\begin{array}{c}
\overrightarrow{i_{3}} \\
\overrightarrow{j_{3}} \\
\overrightarrow{k_{3}}
\end{array}\right)= \\
\left(\begin{array}{ccc}
\cos \omega t \cos \alpha & -\sin \omega t & \cos \omega t \sin \alpha \\
\sin \omega t \cos \alpha & \cos \omega t & \sin \omega t \sin \alpha \\
-\sin \alpha & 0 & \cos \alpha
\end{array}\right) \\
\left(\begin{array}{ccc}
\cos \omega t & \sin \omega t & 0 \\
-\sin \omega t & \cos \omega t & 0 \\
0 & 0 & 1
\end{array}\right) \cdot\left(\begin{array}{l}
1 \\
0 \\
0
\end{array}\right) \\
\left(\begin{array}{c}
\overrightarrow{i_{3}} \\
\overrightarrow{j_{3}} \\
\overrightarrow{k_{3}}
\end{array}\right)=\left(\begin{array}{c}
\cos \alpha \cos ^{2} \omega t+\sin ^{2} \omega t \\
(\cos \alpha-1) \cos \omega t \sin \omega t \\
-\sin \alpha \cos \omega t
\end{array}\right),
\end{gathered}
$$

este resultado representa uma parametrização da hipópede, descrevendo as trajetórias de cada planeta apenas variando o parâmetro $\alpha$ e a velocidade angular definida pelo período sinódico. Na Figura 9 podemos observar a curva paramétrica da hipópede produzida pela equação (30). Também é importante mencionar que a trajetória mostrada na Figura 9 corresponde à trajetória do planeta com respeito ao sistema de vetores unitários 3, sistema da terceira esfera, conforme a nomenclatura da figura 8. Adicionalmente, se pode observar que a figura 
da hipópede tem uma variação de longitude e latitude, a esse respeito Forbes 22$]$ mostrou, usando métodos de trigonometria esférica e expansão em séries, que para o modelo de Eudoxo a variação em latitude é menor que a decima parte da variação em longitude. Na Figura este resultado de Forbes também pode ser verificado.

\section{Discussão e Comentários}

O modelo de Eudoxo reconstruído no século XIX, incluindo as contribuições de Neugebauer no século XX, representa a reconstrução clássica do modelo das esferas concêntricas, no entanto, recentemente vários autores têm criticado esta reconstrução mostrando que outras reconstruções podem também ser feitas, em concordância com as fontes históricas escritas de Aristóteles e Simplício.

Neste respeito foi Mendell [6], quem primeiro mostrou, que respeitando os escritos de Aristóteles e Simplício pode-se deduzir outra reconstrução matemática alternativa para o movimento da Lua, especificamente, escolhendo as velocidades de rotação da segunda e terceira esferas de forma conveniente pode-se construir um modelo alternativo. Neste modelo lunar alternativo se leva em conta que a terceira esfera permite uma explicação do movimento dos nós lunares ${ }^{14}$. No entanto, temos que lembrar que esta interpretação, assim como, a interpretação do século XIX, resultam em base a hipóteses adicionais que temos que assumir em cada caso. Neste sentido ambas reconstruções, a clássica e a alternativa, ficam coerentes quando comparados aos textos históricos. Fica evidente que não temos uma reconstrução única, sendo a principal causa a falta de fontes históricas escritas que possam acotar as hipóteses adicionais que possamos fazer.

Por outra parte, Yavetz 23 mostrou que, de forma similar à reconstrução lunar de Mendell, se pode construir um modelo alternativo para o movimento dos planetas respeitando-se os escritos de Aristóteles e Simplício. Portanto, deve-se tomar maior cuidado com afirmações de textos clássicos como a escrita por Sir Thomas Heath quem menciona que a reconstrução de Schiparelli deve ser considerada como a reconstrução por excelência. Salvo que se conheçam as fontes originais do modelo de Eudoxo, esta afirmação de Heath resulta impertinente.

\footnotetext{
${ }^{14}$ Para uma explicação resumida do mecanismo geométrico do modelo de Mendell revisar a referência 23.
}

As reconstruções apresentadas, de forma pedagógica neste artigo, podem ser consideradas como clássicas 15 mas de fato não são únicas, e levando em conta a matemática moderna das matrizes de rotação resulta mais fácil de apreciar. Ambos modelos permitem calcular em detalhe a figura da hipópede, especialmente o modelo das matrizes que permitem calcular a hipópede de forma paramétrica.

Uma outra questão, recentemente levantada na literatura, em torno do modelo de Eudoxo, se refere a uma afirmação feita por Simplício, na qual afirma que a principal causa para modificar ou substituir o modelo das esferas concêntricas, se refere ao fato deste modelo não poder explicar o aumento de brilho dos planetas, quando eles estão na fase de retrogradação. No entanto, recentemente Carman tem mostrado, do ponto de vista astronômico, como do ponto de vista histórico, que não se têm evidências que mostrem que os planetas aumentam seu brilho na retrogradação, para maiores detalhes revisar o artigo 25.

Por outro lado, alguns pesquisadores são mais radicais nas suas críticas, por exemplo, Bowen [26], tem proposto que a reconstrução clássica do século XIX não pode ser considerada como correta, pois segundo ele as fontes escritas não mostram que os gregos no século IV a.C tenham tido conhecimento do movimento retrógrado dos planetas. Isto mostra que as pesquisas históricas em torno do primeiro modelo da astronomia ainda está longe de ser um tema fechado 16

Neste trabalho foi discutido de forma extensa o modelo de Eudoxo mostrando suas implicações históricas, assim como, sua reconstrução matemática organizada por pesquisadores do século XIX. Este modelo constitui o primeiro modelo matemático da história da astronomia e é notável destacar a interação entre a matemática e as observações como inspiração de formulação do modelo, sendo esta uma característica fundamental das ciências até agora. Pelos documentos históricos não sabemos qual era a proposta matemática original de Eudoxo, também não sabemos quais eram sua ideias sobre a realidade das esferas, é possível que devido a sua formação matemática

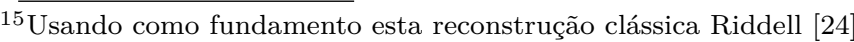
discute a construção da hipópede e outras construções matemáticas atribuídas a Eudoxo como, por exemplo, a duplicação do cubo.

${ }^{16}$ Para uma maior discussão sobre recentes aspectos desta teoria revisar a referência 15 .
}

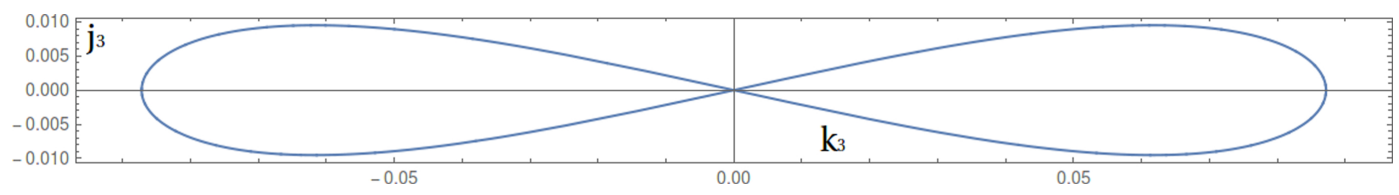

Figura 9: Figura construída mediante o programa Mathematica utilizando a equação (30). Adotou-se uma inclinação $\alpha$ de 5 e o período sinódico do planeta Júpiter. Na horizontal consideramos a longitude, a qual fazemos coincidir com a eclíptica, isto é, o eixo $k_{3}$, na vertical, latitude, consideramos o eixo $j_{3}$, a profundidade dada pelo eixo $i_{3}$ tomamos como desprezível. Também se observa que a escala vertical (latitude) é aproximadamente uma decima da escala horizontal (longitude), isto é, o resultado de Forber [22]. 
Eudoxo ficasse interessado em uma solução matemática engenhosa, sem ter preocupações subjacentes associadas com ideias metafísicas. Do ponto de vista da correspondência entre as predições do modelo e as observações astronômicas é notável mencionar que para os planetas como Júpiter e Saturno se consiga mostrar uma correspondência qualitativa [1].

Neste artigo tentamos dar uma ideia completa do modelo de Eudoxo, tanto na discusão das fontes históricas, como na reconstrução matemática. Esperamos que este artigo seja de utilidade para todos os que queram se aprofundar na história da astronomia grega e possa servir de material para introduzir discussões sobre história e evolução da ciência.

\section{Agradecimentos}

A.M.V.T. gostaria de agradecer à UFES por permitir coordenar um projeto de pró-ensino da Pró-reitoria de graduação e M.O gostaria de agradecer a bolsa concedida pelo projeto de pró-ensino da UFES. Este trabalho foi iniciado dentro deste programa.

\section{Referências}

[1] O. Neugebauer, A history of ancient mathematical astronomy (Springer Science \& Business Media, Rhode lsland, 1975), v. 1, p. 1468.

[2] O. Neugebauer,Astronomy and history selected essays (Springer Science \& Business Media, New York, 1983), v. 1, p. 553 .

[3] C.C. Carman and J. Evans, Archive for History of Exact Sciences 6, 68 (2014).

[4] A. Koyre, Estudos de historia do pensamento científico (Ed. Forense Universitaria, São Paulo, 1982), v. 1, p. 200.

[5] E.D. Sylla, The Oxford Calculators and the mathematics of motio (Garland Publishing, Oxford, 1849), p. 1320.

[6] H. Mendell, Centaurus 40, 177 (1998).

[7] J.L.M. Dreyer, A history of astronomy from Thales to Kepler (Courier Corporation, London, 1953), v. 2 , p. 465.

[8] Platão, Timeu-Critías (Centro de Estudos Clássicos e Humanísticos, Coimbra, 2011).

[9] D. Panchenko, Journal for the History of Astronomy 4, 25 (1994).

[10] D. Proust, International Astronomical Union 5, S260 (2009).

[11] Aristotle e W.D.Ross, Metaphysics (Clarendon Press, Oxford, 1924), p. 368.

[12] S. Weinberg, Para explicar o mundo: A descoberta da ciência moderna (Companhia das Letras, São Paulo, 2015), v. 1, p. 509.

[13] D.R. Dicks, Early Greek astronomy to Aristotle (Thames and Hudson, London, 1971).

[14] G.E.R. Lloyd, Aristotelian Explorations (Cambridge University Press, Cambridge, 1996).

[15] A. Gregory e V. Trimble, History of Astronomy 31, 3 (2000).
[16] T. Heath, Aristarchus of Samos: the ancient Copernicus (Courier Corporation, Chelmsford, 2004).

[17] J.C. Schaubach, Geschichte der griechischen Astronomie bis auf Eratosthenes (Göttingen, Röwer, 1802).

[18] L. Ideler, Abhandlungen der Königlichen Akademie der Wissenschaften in Berlin 1, 189 (1828)

[19] E.F. Apelt, Die Sphärentheorie des Eudoxus und Aristoteles (W. Engelmann, São Paulo, 1849).

[20] G.V. Schiaparelli, Scritti sulla storia della astronomia antica (Mimesis Edizioni, Milano, 1997), p. 35.

[21] C.M. Linton, From Eudoxus to Einstein: a history of mathematical astronomy (Cambridge University Press, New York, 2004), v. 1, p. 503.

[22] E.G. Forbes, British Astronomical Association 83, 196 (1973).

[23] I. Yavetz, Archive for History of Exact Sciences 3, 52 (1998).

[24] R.C. Riddell, Archive for History of Exact Sciences 1, 20, (1979).

[25] C.C. Carman, Studies in History and Philosophy of Science 54, 90 (2015).

[26] A.C. Bowen, Perspectives on Science 2, 10 (2002). 